\title{
A Group-Based Architecture for Wireless Sensor Networks
}

\author{
Jaime Lloret, Miguel Garcia and Jesus Tomas \\ Department of Communications \\ Polytechnic University of Valencia \\ Valencia, Spain \\ jlloret@dcom.upv.es, migarpi@teleco.upv.es,jtomas@dcom.upv.es
}

\begin{abstract}
Many routing protocols for ad-hoc networks and sensor networks have been designed, but none of them are based on groups. We propose to divide the network into several groups of sensors. When a sensor send data to other groups, the data has to arrive just to one sensor from each group, then they propagate it to the rest of sensors in their groups. We have simulated our proposal for different types of sensor topologies to know which type of topology is the best depending on the number of sensors in the whole network or depending on the number of interior sensors. We have also simulated how much time is needed to propagate information between groups. The application areas for our proposal could be rural and agricultural environments to detect plagues and to propagate it to neighbouring areas, or for military purposes to propagate information between neighbouring squads.
\end{abstract}

Keywords-Sensor Network; Group-Based Architecture; Groupbased routing algorithm.

\section{INTRODUCTION}

There are many routing protocols that can be applied to sensor networks. They can be classified into two groups [1] [2]. One group is formed by protocols based on the network topology and the other group the ones that do not take it into account. First group can be broken down into three subgroups:

1-Plane routing. All nodes in the network have the same role and perform the same tasks. Because of the number of nodes in these networks, the use of a global identifier, for every node, is not feasible. It uses a data-centric routing where the base station sends requests to some regions and the nodes from that regions reply. Some of the algorithms in this group are SPIN, Direct diffusion, Rumour routing, MCFA, GBR, IDSQ, CADR, COUGAR, ADQUIRE, and so on.

2 -Hierarchical routing. It is very scalable and has an efficient communication. It has been designed for energy saving purposes, because central nodes have unlimited energy, while leaf sensors have limited energy. When the sensor network topology is formed, data can be routed. Some algorithms such as LEACH, PEGASIS, TEEN, APTEEN, MECN, Virtual grid architecture routing and TTDD are hierarchical routing algorithms.

3-Position-based routing. All data is routed through the sensors depending on their position. Distances between sensors are known because of neighbouring sensors signals. There are other protocols that base node's situation on GPS and, using that information, route the data to the most adequate sensor. These algorithms consume more energy than others because of the need of GPS signal. Some of those algorithms sleep sensors when the network has not any activity. Some examples are GAF, GEAR, GOAFR and SPAN.

Second group does not have into account the structure of the network. It can be broken into five subgroups:

1-Multipath Routing Protocols. The information could reach the destination through different paths. Because sensors have to calculate several paths, they use a main route when they have enough energy; otherwise, they use an alternative path.

2-Query-Based Routing protocols. They are based on a central node that sends a query about an event to the specific area. When the query arrives to that area, it is routed to the destination sensor, and then it will reply. A sensor from an area could be sleeping, saving energy, while there is not any query to that area.

3-Negotiation-Based Routing Protocols. Before data transmission, the sensor has to negotiate the data it has to send, so redundant data could be deleted, and resources will be available while data exchange. SPIN protocols use this type of routing, but they take into account the network structure.

4-QoS protocols. The information is routed to the sensors taking into account quality parameters such as delay, energy, bandwidth and so on. SAR and SPEED protocols are based on quality of service algorithms.

5-Data coherent/incoherent processing based protocols. These algorithms use several routing techniques taking into account the data processing of a coherent or incoherent result.

None of the routing protocols aforementioned are groupbased. We propose to divide the network of sensors into several groups and if a sensor has to send data to other groups, when this data arrives to one sensor from a group, it propagates it to the rest of sensors in its group.

The paper is structured as follows. Section 2 examines some works related with our proposal such as neighbour selection and architectures based on groups, and explains our motivation. There is a description of our architecture proposal in section 3. Analytical model for some types of topologies of sensors are shown in section 4 . The propagation time to reach a sensor from other group is analyzed in section 5. Finally, section 6 gives our conclusions and future works. 


\section{PREVIOUS WORKS AND MOTIVATION}

Throughout the years, different types of strategies for neighbors' selection have been developed. On one hand, there are the ones used for transfer coordination to increase content availability. They can be applied for P2P networks [3], [4] and [5], for content delivery systems [6] or for distributing systems [7]. Many other systems locate nodes in the topology based on mathematical structures such as CAN, Chord, Pastry and Tapestry, but these systems do not take care of the underlying network, so a neighbor of a node could be very far (in terms of round-trip time -RTT-) or it could not have enough capacity available to perform its necessities. There are proposals where nodes' connections are based on the underlying network, such as Plethora [8] or on their geographic location such as the one described in [9]. Other systems locate new nodes in the topology taking into account that they are possibly close to a given node, and then, perform RTT measurements to identify the actual closest node such as the one presented in [10], and others use a proximity neighbor selection (PNS) using heuristics approximations such as the one presented in [11]. There are also some researches for wireless networks, where connections are established only if they are closed, because of their coverage area ([12] and [13]).

But none of the neighbor selection strategies shown consider to group nodes and structure connections between nodes from different groups. On the other hand, none of them take into account the capacity of the nodes to select the neighbor to have a connection with.

There are several works in the literature where nodes are grouped into groups and connections are established between nodes from different groups, but all of them have been developed to solve specific issues. Rhubarb [14] organizes nodes in a virtual network, allowing connections across firewalls/NAT, and efficient broadcasting. The nodes can be active, if they establish connections, or passive, if they don't. Rhubarb system has only one coordinator per group and coordinators could be grouped in groups in a hierarchy. The system uses a proxy coordinator, an active node outside the network, and all nodes inside the network make a permanent TCP connection with the proxy coordinator, which is renewed if it is broken by the firewall or NAT. If a node from outside the network wishes to communicate with a node that is inside, it sends a connection request to the proxy coordinator, who forwards the request to the node inside the network. Rhubarb has a three-level group's hierarchy. It may be sufficient to support a million nodes but when there are several millions of nodes in the network it could not be enough, so it suffers from scalability problems. On the other hand, all nodes need to know the IPs of the proxy coordinator nodes to establish connections with nodes from other virtual networks. A Peer-to-Peer Based Multimedia Distribution Service has been presented in [15]. That paper proposes a topology-aware overlay in which nearby hosts or peers self-organize into application groups. End hosts within the same group have similar network conditions and can easily collaborate with each other to achieve QoS awareness. When a node in this architecture wants to communicate with a node from other group, the information is routed through several groups until it arrives to the destination but this solution only can be applied to logical networks because of neighboring nodes could be so far. There are other architectures based on super-peer models such as Gnutella 2 and FastTrack networks. Each super-peer in these networks creates a group of leaf nodes. Superpeers perform query processing on behalf of their leaf nodes. A leaf node sends the query to its superpeer that floods it to its superpeer neighbors up to a limited number of hops. The main drawback of this architecture is that all information has to be routed through the superpeer logical network. Finally, there are some hierarchical architectures were nodes are structured hierarchically and some parts of the tree are grouped into groups such as the ones presented in [16] and in [17]. In some cases, some nodes have connections with nodes from other groups although they are in different layers of the tree, but in all cases, the information has to be routed through the hierarchy to achieve nodes from other groups, so all layers of the hierarchy could be overloaded in case of having many data to be transferred.

Let's suppose we need to divide the network into groups or areas because of the physical implementation of the sensor network or for scalability purposes. All architectures previously shown don't solve that problem efficiently, because in the case of centralized architectures, the server will have many wireless connections at the same time, so it will need many resources. On the other hand, there is a central point of failure and a bottleneck. In the case of fully distributed architectures, it is very difficult to control the system and it needs much time to process tasks, because of the time needed to reach far nodes, decreasing the performance of the whole system.

\section{ARCHITECTURE DESCRIPTION}

Our proposal is based on the creation of groups of sensors with the same functionality in the network. There is a central sensor that limits the zone where the sensors from the same group will be placed, but its functionality will be the same that the rest of the sensors. A sensor knows in which group is because it is given manually or by GPS.

When there is an event in one sensor, this event is sent to all sensors in its group. All nodes in a group know all information of their group. Border sensors are those sensors of the border of the group, and they have connections with border sensors from other groups as it is shown in figure 1.

Border sensors are used to send information to other groups or to receive information from other groups and distribute it inside. When a sensor has to send some information to its group and to neighboring groups, the information is forwarded using Reverse Path Forwarding (RPF) Algorithm [18] (each group has one RPF database), but when the information has to be sent to other groups only, the information is routed directly to the border sensor closest to that group. When the sensor from the neighbor group receives that information, it routes it to all nodes in its group. Because the system is based on groups, the information is forwarded very fast to other groups (the information is routed through the shortest path to the border area sensor). Connections between border sensors from different groups are established as a function of their available processing capacity, their available number of connections, their available power or because a neighbor sensor failure. Figure 2 shows a logical view of the proposed architecture. 


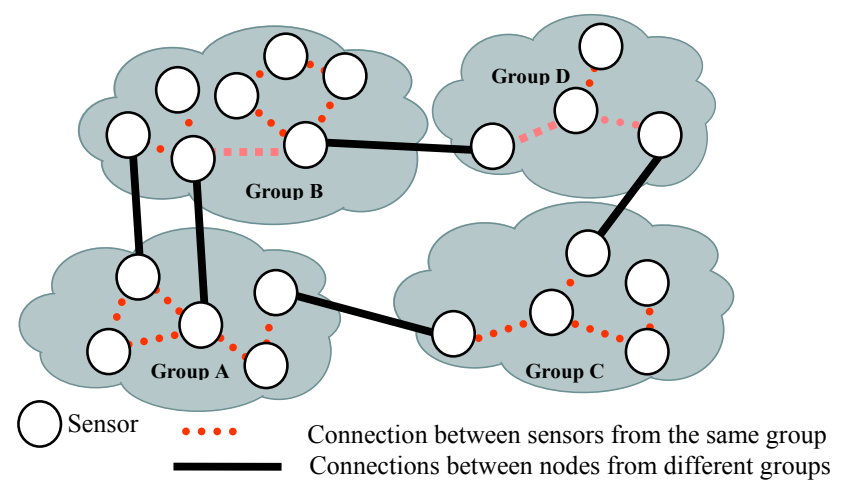

Figure 1. Topology example

\section{ANALITICAL MODEL}

This section describes the architecture analytically taking into account that it is a system based on groups. Now, we are going to analyze the architecture for several types of network architectures inside the groups.

Let a network of sensors $\mathrm{G}=(V, \lambda, E)$ be, where $V$ is the set of sensors, $\lambda$ is the set of their capacities $(\lambda(i)$ is the capacity of the $i$-th sensor and $\lambda(\mathrm{i}) \neq 0 \forall \mathrm{i}$-th sensor) and $E$ is the set of connections between sensors. Let $k$ be a finite number of disjoint subsets of $V$, so $V=\cup V_{k}$ and there is not any sensor in two or more subsets $\left(\cap V_{k}=0\right)$. Let's suppose $\mathrm{n}=|\mathrm{V}|$ (the number of sensors in $V$ ) and $k$ the number of subsets of $V$. We obtain equation 1 .

$$
n=\sum_{i=1}^{k}\left|V_{k}\right|
$$

Every $V_{k}$ has a central sensor, several intermediate sensors and several border sensors as it is shown in expression 2 .

$$
n=1+n_{\text {intermediate }}+n_{\text {border }}
$$

Now we can describe the whole network as the sum of all these sensors from all groups as it is shown in equation 3 .

$n=\sum_{i=1}^{k}\left|\left(n_{\text {central }}+n_{\text {intermediate }}+n_{\text {border }}\right)_{k}\right|=k+\sum_{i=1}^{k}\left(\mid n_{\text {intermediat }}\right)_{k}+\sum_{i=1}^{k}\left(\mid n_{\text {border }}\right)_{k}$

Now we are going to model our proposal as a function of the number of intermediate and border sensors in a network for several types of networks.

\section{A. Tree topology}

Tree topologies have a sensor acting as a trunk and from this sensor leaves several branches. There are two types of tree topologies: N-nary trees (every sensor has the same number of leaf nodes, binary, ternary and so on) and backbone trees, where there is a trunk and there are sensors that branch from it. In both cases the information flows hierarchically. We are going to study the first case only, because it could be easily implemented by limiting the number of incoming connections in a sensor. The backbone tree is a special case of the partially centralised P2P Networks with superpeers and it will be discussed later.
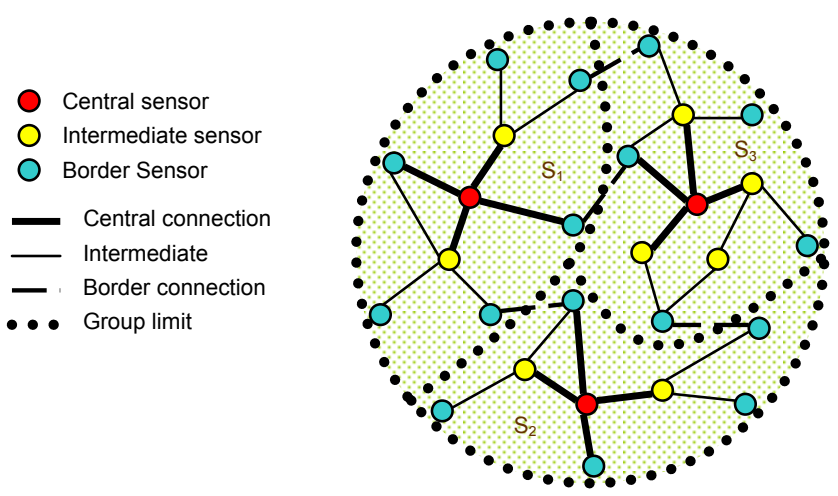

Figure 2. Logical view of the proposed architecture

In a tree topology, the number of sensors $n$ is equal to $\mathrm{M}^{\mathrm{k}}$ 1 , where $M=2$ in case of a binary tree, $M=3$ in a ternary tree and so on, and $k$ is the number of levels of the tree). The number of links is $n-1$ and the diameter of the network is $2 \bullet k-2$. We suppose balanced trees where all branches have the same number of levels, so the number of intermediate sensors is given by expression 4 .

$$
n_{\text {int ermediate }}=\frac{n-1}{\text { grade }}-1
$$

Where grade is the number of leaf sensors for each sensor. Using expressions 2 and 4, we obtain expression 5. It gives the number of border sensors related with the number of intermediate sensors.

$$
n_{\text {border }}=(\text { grade }-1) \bullet n_{\text {intermediate }}+\text { grade }
$$

Tree topologies have been implemented in several sensor networks such as the one shown in [19].

\section{B. Grid topology}

We are going to consider 2-dimensional Grid and 3dimensional Grid with all its sides equals. To make easy the mathematical development, in a 2D Grid we will use a square matrix where $n=m$ for $n \geq 3$ and in a 3D Grid we will use a cube matrix where $n=m=l$ for $n \geq 3$. In both cases, the case of $n=3$ has one central sensor, but there is not any intermediate sensor.

The number of sensors in a 2D Grid, with all sides equals, sensor network is $n^{2}(n=3,4 \ldots)$. The number of neighbours of an intermediate sensor is 4 , the border sensor has 3 neighbours and the vertex sensor has 2 neighbours. The number of connections in the topology is given by expression 6 .

$$
l=2 \cdot(n-\sqrt{n})
$$

Expression 7 gives the diameter of a 2D Grid topology.

$$
d=2 \cdot(\sqrt{n}-1)
$$

We have observed that the number of border sensors in a 2D Grid topology follows the expression 8.

$$
n_{\text {border }}=4 \cdot(\sqrt{n}-1)
$$

Using expression 2, we obtain expression 9. It gives the number of intermediate sensors.

$$
n_{\text {intermediate }}=n-4 \cdot(\sqrt{n}-1)-1
$$


Using expressions 8 and 9, we obtain expression 10 that relates the number of border sensors related with the number of intermediate sensors.

$$
n_{\text {border }}=4 \cdot\left(1+\sqrt{n_{\text {int ermediate }}+1}\right)
$$

2D Grid topologies have been implemented in several sensor works such as the one shown in [20].

The number of sensors in a 3D Grid, with all sides equals, sensor network is $\mathrm{n}^{3}(\mathrm{n}=3,4 \ldots)$. The number of neighbours of an intermediate or central sensor is 6 , border sensors have 5 neighbours and the vertex sensors have 4 neighbours. Expression 11 gives the number of connections in the topology.

$$
l=3 \cdot\left(n-\sqrt[3]{n^{2}}\right)
$$

Expression 12 gives the diameter of a 3D Grid topology.

$$
d=3 \cdot(\sqrt[3]{n}-1)
$$

The number of border sensors in a 3D Grid topology can be measured by expression 13 .

$$
n_{\text {border }}=6 \cdot \sqrt[3]{n^{2}}-12 \cdot \sqrt[3]{n}+8
$$

Using equation 2 , we can obtain the number of intermediate sensors in a 3D Grid topology.

$$
n_{\text {int ermediate }}=n-6 \cdot \sqrt[3]{n^{2}}+12 \cdot \sqrt[3]{n}-9
$$

Using the cube geometry, we can obtain the number of border sensors as a function of the number of intermediate sensors. This relation is given by equation 15 .

$$
n_{\text {border }}=6 \cdot \sqrt[3]{\left(n_{\text {int ermediate }}+1\right)^{2}}+12 \cdot \sqrt[3]{\left(n_{\text {int ermediate }}+1\right)}+8
$$

3D Grid topology is used in networks that need many paths to reach the same destination.

\section{Power Law}

In [21], M. Faloutsos et al. show that the nodes of a distribution network can be modelled using mathematical laws. This paper states that power law fits real measurements with correlation coefficients of $96 \%$. Power law states that the grade of a node $\left(d_{v}\right)$ is proportional to its range $\left(r_{v}\right)$ to the power of a constant called $\mathrm{R}$ as it is shown in expression 16.

$$
d_{v} \propto r_{v}^{R}
$$

Where $\mathrm{R}$ varies depending on it is applied. Applying Lemma 1, from paper [21], the grade of a node is given by expression 17.

$$
d_{v}=\frac{1}{n^{R}} \cdot r_{v}^{R}
$$

Where $n$ is the number of sensors in the network, $d_{v}$ and $r_{v}$ are the grade and the range of the $v$ sensor respectively.

From the power law appears the Zipf's law. It states that some nodes have many links while many nodes have one or two links. Zipf's law has been proposed by B. A. Huberman et al. to model Internet in [22], and by Z. Ge et al. to model Gnutella and Napster Networks in [23].
Zipf 's function states that the range of $r$ nodes follows the proportionality shown in expression 18 .

$$
f(r)=C \cdot r^{-\alpha}
$$

Where $\alpha$ varies depending on the type of distribution of the nodes. It is also known as the Zipf coefficient. $\mathrm{C}$ is a constant that varies depending on the type of network.

Taking into account expressions 17 and 18, we can assume that $\mathrm{R}=-\alpha$. Applying Zipf's law to our sensor architecture, we obtain expression 19.

$$
n_{\text {border }}=\frac{n^{\alpha}}{\left(n_{\text {int ermediate }}+1\right)^{\alpha}}
$$

Taking expression 2 into account, we can obtain expression 20. It relates the number of border sensors with the total number of sensors in the topology.

$$
n=\frac{\left(n_{\text {border }}\right)^{\frac{\alpha+1}{\alpha}}}{\left(n_{\text {border }}\right)^{1 / \alpha}+1}
$$

On the other hand, replacing expression 2 in expression 19 we obtain the number of border sensors as a function of the number of intermediate sensors as it is shown in expression 21.

$$
n_{\text {border }}=\frac{\left(n_{\text {border }}+n_{\text {int ermediate }}+1\right)^{\alpha}}{\left(n_{\text {int ermediate }}+1\right)^{\alpha}}
$$

As Internet topology has varied along the years, because of the growth of the number of computers connected to it, $\alpha$ value has varied from 0.74 to 3 in last measures, as it can be seen in [21] and [24].

\section{Logarithmic law}

Logarithmic law was introduced by György Hermann in [25]. This law proposes that the border nodes, or the nodes with higher roles in the network, are the responsible of the stability of the network. It also proposes that the border nodes are the responsible of the security of the network because they are the ones that communicate with exterior nodes. This proposal follows the model developed by D. J. Watts et al. in [26], where connections are established based on efficiency, stability and security features.

This law states that the distance between two border sensors is given by expression 22 .

$$
l_{n} \approx l_{\text {max }} \approx \ln \left(n_{\text {int ermediate }}+1\right)
$$

Where $l_{\max }$ is the diameter of the network. It is equal to the logarithm of the nodes that don't are in the border of the network (the central sensor plus the intermediate sensors).

The relationship between the number of border sensors and the intermediate sensors is given by expression 23 .

$$
c \cdot\left(n_{\text {int ermediate }}+1\right) \leq n_{\text {border }} \leq c \cdot\left(n_{\text {int ermedate }}+1\right) \cdot \ln \left(n_{\text {int ermedate }}+1\right)
$$

$C$ is a constant that depends on the model of the network.

So, the number of sensors in the network is set between limits shown in equation 24 . 
$\begin{cases}n_{\text {border }}=\frac{n}{2} & \text { When } \mathrm{n}_{\min } \\ n=\left(n_{\text {int ermediate }}+1\right) \cdot\left(1+\ln \left(n_{\text {int ermediate }}+1\right)\right) & \text { When } \mathrm{n}_{\max }\end{cases}$

\section{E. Partially centralized P2P networks}

In [27], J. Lloret et al. proposed an architecture for partially centralized P2P networks. They measured the number of brokers or superpeers (depending on the type of network), that was inside the architecture on behalf of all brokers or superpeers in the whole network. Those values could be applied to the proposal presented in this paper if we suppose that the intermediate sensors plus the central one are the distribution nodes and the border sensors are the nodes considered in the access layer. The relationship between intermediate sensors and border sensors are different according on the type of P2P network as it is shown in expression 25.

$\mathrm{n}_{\text {border }}= \begin{cases}n_{\text {int ermediate }}+1 & \text { in a broker model } \\ 96 \cdot\left(n_{\text {int ermediate }}-1\right) & \text { in a superpeer model }\end{cases}$

Using expression 2 we obtain expression 26.

$\mathrm{n}_{\text {border }}= \begin{cases}\frac{n}{2} & \text { in a broker model } \\ \frac{96 \cdot(n-2)}{97} & \text { in a superpeer model }\end{cases}$

\section{F. Architectures comparation.}

This section compares the number of border sensors versus the number of intermediate sensors and the number of border sensors versus the number of sensors in the group for all architectures shown. In both cases, partially centralized P2P networks with brokers model is the same case than the minimum values of the logarithmic model.

Figure 3 graphs the number of border sensors in the group as a function of the number of intermediate sensors for all models previously analyzed. For Zipf's law we have used numerical methods to obtain its graph. In figure 6, we can observe that if a group with few border sensors is needed, if there are less than 24 intermediate sensors, the best election is the minimum value of the logarithmic law, but if we have more than 24 intermediate sensors the best one is $2 \mathrm{D}$ Grid. What is desirable is to have many border sensors in order to have many connections with sensors from other groups, so there will be higher probability to contact with more neighbouring groups. We have checked that for less than 770 intermediate sensors the best topology is the partially centralized P2P networks with superpeer model, but if the number of intermediate sensors is equal or higher that 770, the best topology is Zipf's law with $\mathrm{R}=-2.45$.

Figure 4 shows the number of border sensors in the group as a function of the number of sensors in the group. We have used numerical methods to know the number of border sensors as a function of the number of sensors in the group for the logarithmic model and for Zipf's law. In figure 4, we can observe that, when many border sensors are needed versus the number of sensors in the group, for less than 40 sensors the best election is $3 \mathrm{D}$ Grid, but for 40 sensors or more, the best election is the partially centralized network with superpeers model. When we need few border sensors versus the number of sensors in the group, for less than 110 sensors the best topology is the ternary tree, but for more than 110 sensors the best topology is $2 \mathrm{D}$ Grid.

\section{Propagation Time}

Every time a sensor has to send information to a specific group, first it has to send the information to the border sensor closest to that group, and then, the information has to be sent through the groups till the information arrives to the destination group. Expression 27 formulates it mathematically.

$$
T=t_{\text {to_ border }}+\sum_{i=1}^{n} t_{\text {max }_{-} \text {int ragroup }} i+\sum_{i=1}^{n+1} t_{\text {border }_{-} i-\text { border }}{ }_{-i+1}
$$

Where $t_{\text {to_border }}$ is the time needed to reach the border sensor closest to that group, $n$ is the number of intermediate groups through that path, $t_{\text {max_intragroup_ }} i$ is the time needed to cross the $\mathrm{i}$ th group and $t_{\text {border }-i \text {-border } i+1}$ is the time needed to transmit the information from one border sensor to another border sensor from other group.

Let's suppose that $t_{p}$ is the mean value of the propagation time for all transmissions between 2 sensors in the architecture. So, we can assume that $t_{\text {border } \_ \text {- } \text {-border }_{i+1}}=t_{p}$ and, given $d_{1}$ hops to reach from a source sensor to the border sensor closest to the destination group, we can assume $t_{\text {to__order }}=d_{I} \cdot t_{p}$. We can define the time needed to cross the $\mathrm{i}^{\text {th }}$-group as $t_{\text {max }}$ intragroup $i=d_{i} \cdot t_{p}$, where $d_{i}$ is the number of hops to cross the $\mathrm{i}^{\text {th }}$-group. Expression 28 gives the time needed to reach a group as a function of the mean value of the propagation time.

$$
T=\left(d_{1}+\sum_{i=1}^{n} d_{i}+n+1\right) \cdot t_{p}
$$

Let's consider four groups along a path to a group destination. Figure 5 shows two simulations. The first one (source group) shows the time needed when the mean value of the number of hops to cross the groups involved in the path is 10 and the number of hops from the source sensor to the border sensor closest to the destination group vary from 1 to 32 . The second one (mean value of groups) shows the time needed when the number of hops from the source sensor to the border sensor closest to the destination group is 10 and the mean value of the number of hops to cross the groups involved in the path vary from 1 to 32 . In figure 5, we can observe that the delay is higher when the mean value of the number of hops in the groups increases, but it is less significant when the number of hops from the source sensor to the border sensor closest to the destination group increases.

\section{CONCLUSIONS}

To the extent of our knowledge, there is not any previous interconnection system to structure connections between groups of nodes like the one presented in this paper. This paper demonstrates that it is a feasible option and it is independent of the structure of the sensors of the group, but some group architectures perform better than others. It could be applied to specific environments such as rural environments or for military purposes. We are now designing its fault-tolerance. 


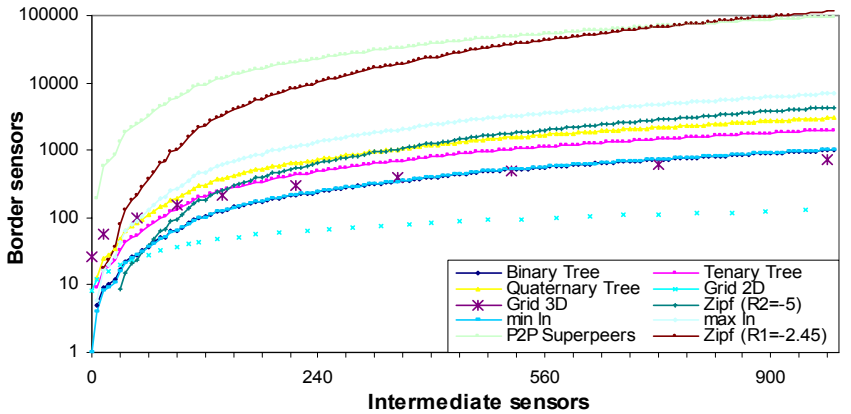

Figure 3. Border sensors as a function of the intermediate sensors

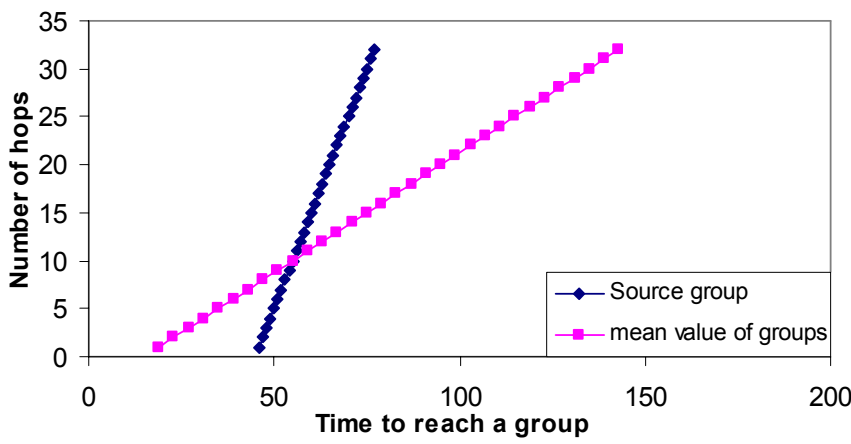

Figure 5. Time to reach a group as a function of the number of hops

\section{ACKNOWLEDGMENT}

Authors thank Mr. Antonio Canos, from the Polytechnic University of Valencia, because of his support obtaining, using numerical methods, the inverse of some functions used here.

\section{REFERENCES}

[1] L.M. Feeney, A taxonomy for routing protocols in mobile ad hoc networks, October 1999, SICS Technical Report T99:07.

[2] J. N. Al-Karaki, A. E. Kamal, Routing techniques in wireless sensor networks: a survey. IEEE Wireless Communications Volume 11, Issue 6, pp. 6-28, Dec. 2004.

[3] L. Zou, E. Zegura, M.H. Ammar, The effect of peer selection and buffering strategies on the performance of peer-to-peer file sharing systems, in: Proceedings of Tenth International Symposium on Modeling, Analysis and Simulation of Computer and Telecommunication Systems, MASCOTS, Fort Worth, TX, 2002.

[4] Simon G.M. Koo, Karthik Kannan and C.S. George Lee, On neighborselection strategy in hybrid peer-to-peer networks, Future Generation Computer Systems 22 (7) (2006). Pp. 732-741.

[5] D.S. Bernstein, Z. Feng, B.N. Levine, S. Zilberstein, Adaptive peer selection. Proceedings of the 2nd International Workshop on Peer-toPeer Systems, IPTPS03, Berkeley, CA, February 2003.

[6] J. Byers, J. Considine, M. Mitzenmacher, S. Rost, Informed content delivery across adaptive overlay networks, in: Proceedings of ACM SIGCOMM 2002, Pittsburgh, PA, August 2002.

[7] S.G.M. Koo, C.S.G. Lee, K. Kannan, A resource-trading mechanism for efficient distribution of large-volume contents on peer-to-peer networks, Proc. of the 14th IEEE International Conference on Computer Communications and Networks, San Diego, CA, October 17-19, 2005, pp. $428-433$.

[8] Ferreira, R. A., Jagannathan, S., Grama, A. Locality in structured peerto-peer networks, Journal of Parallel and Distributed Computing, Vol. 66, Issue 2. Feb. 2006. Pp. 257-273.

[9] Bose, P., Morin, P., Stojmenovic, I., Urrutia, J. Routing with guaranteed delivery in ad hoc wireless networks, Wireless Networking 7 (6) (2001). Pp. 609-616.

[10] Xu, Z., Tang, C., Zhang, Z. Building topology-aware overlays using global soft-state. Proc. of the 23rd International Conference on Distributed Computing Systems, 2003. May 2003.

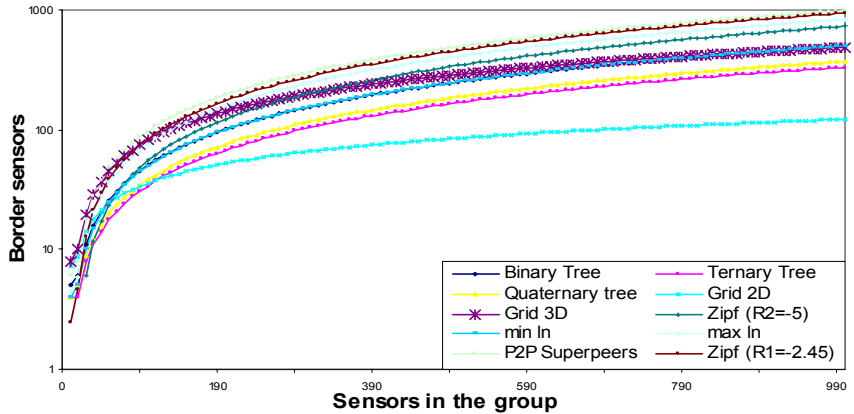

Figure 4. Border sensors as a function of the number of sensors in the group

[11] Castro, M., Druschel, P., Hu, Y. C. and Rowstron, A. Proximity neighbor selection in tree-based structured peer-to-peer overlays, Technical Report MSR-TR-2003-52, Microsoft Research, Microsoft Corporation. 2003.

[12] Karp, B., Kung, H.T. GPSR: greedy perimeter stateless routing for wireless networks, in: Proceedings of the Sixth Annual International Conference on Mobile Computing and Networking (MobiCom '00), ACM Press. New York, NY, USA, 2000, Pp. 243-254.

[13] Kim, Y. J., Govindan, R., Karp, B., Shenker, S. Geographic routing made practical, in: Proceedings of the Second USENIX/ACM Symposium on Networked System Design and Implementation (NSDI'05), May 2005, pp. 217-230.

[14] Wierzbicki, A., Strzelecki, R., Swierczewski, D. and Znojek, M. Rhubarb: a Tool for Developing Scalable and Secure Peer-to-Peer Applications, in: Second IEEE International Conference on Peer-to-Peer Computing (P2P2002), Linöping, Sweden, 2002.

[15] Xiang, Z., Zhang, Q., Zhu, W., Zhang, Z. and Zhang, Y. Peer-to-Peer Based Multimedia Distribution Service, IEEE Transactions on Multimedia 6 (2) (2004)

[16] Hongjun, L., Luo, L. P. and Zhifeng, Z. A structured hierarchical P2P model based on a rigorous binary tree code algorithm, Future Generation Computer Systems 23 (2). 2007. Pp. 201-208.

[17] Thallner, B., Moser, H. Topology control for fault-tolerant communication in highly dynamic wireless networks. Proceedings of the Third International Workshop on Intelligent Solutions in Embedded Systems. May 2005.

[18] Yogen K. Dalal y Robert M. Metcalfe, "Reverse path forwarding of broadcast packets", Communications of the ACM. Volume 21, Issue 12 Pp: 1040 - 1048. Diciembre 1978. I. S. Jacobs and C. P. Bean, "Fine particles, thin films and exchange anisotropy," in Magnetism, vol. III, G. T. Rado and H. Suhl, Eds. New York: Academic, 1963, pp. 271-350.

[19] Jan Beutel, Matthias Dyer, Martin Hinz, Lennart Meier and Matthias Ringwald. Next-generation prototyping of sensor networks, Proceedings of the 2nd international conference on Embedded networked sensor systems. Pp: 291 - 292. 2004.

[20] Radu Stoleru and John A. Stankovic, Probability Grid: A Location Estimation Scheme for Wireless Sensor Networks, Sensor and Ad Hoc Communications and Networks, 2004. IEEE SECON 2004. Pp. 430438. 4-7 Oct. 2004

[21] Siganos, G., Faloutsos, M., Faloutsos, P. and Faloutsos, C. Power Laws and the AS-Level Internet Topology. IEEE/ACM Transactions on Networking, Vol. 11, Issue 4. August 2003.

[22] Huberman, B. A. and Adamic, L. A. Growth dynamics of the WorldWide Web, Nature, vol. 40, (1999). Pp. 450-457.

[23] Ge, Z., Figueiredo, D. R., Jaiswal, S., Kurose, J., Towsley, D. Modeling Peer-Peer File Sharing Systems, Proceedings IEEE INFOCOM 2003, San Francisco, March-April 2003.

[24] Albert, R., Jeong, H. \& Barabási, A. L. Diameter of the World Wide Web. (1999) Nature 401, 130-131.

[25] Gyorgy Hermann. Mathematical investigations in network properties. Proceedings IEEE Intelligent Engineering Systems, 2005. INES '05. Pp 79-82 September 16-19, 2005

[26] D. J. Watts and H. S. Strogatz. Collective dynamics of 'small-world' networks. Nature 393, 440 (1998).

[27] Lloret, J., Boronat, F., Palau, C., Esteve, M.: Two Levels SPF-Based System to Interconnect Partially Decentralized P2P File Sharing Networks, Joint International Conf. on Autonomic and Autonomous Systems International Conf. on Networking and Services (2005). 